OPEN ACCESS

Edited by:

Victoria Team

Monash University, Australia

Reviewed by:

Lilian Perez,

RAND Corporation, United States Shamshad Khan

University of Texas at San Antonio,

United States

Michaela Pascoe,

Victoria University, Australia

*Correspondence:

Guy Faulkner

guy.faulkner@ubc.ca

Specialty section

This article was submitted to Health Communication,

a section of the journal

Frontiers in Communication

Received: 12 June 2020

Accepted: 17 August 2020

Published: 23 September 2020

Citation:

Faulkner G, Rhodes RE,

Vanderloo LM, Chulak-Bozer T,

O'Reilly N, Ferguson L and Spence JC (2020) Physical Activity as a Coping

Strategy for Mental Health Due to the

COVID-19 Virus: A Potential

Disconnect Among Canadian Adults?

Front. Commun. 5:571833

doi: 10.3389/fcomm.2020.571833

\section{Physical Activity as a Coping Strategy for Mental Health Due to the COVID-19 Virus: A Potential Disconnect Among Canadian Adults?}

\author{
Guy Faulkner 1*, Ryan E. Rhodes², Leigh M. Vanderloo 3,4 , Tala Chulak-Bozer ${ }^{4}$, \\ Norm O'Reilly ${ }^{5}$, Leah Ferguson ${ }^{6}$ and John C. Spence ${ }^{7}$
}

'School of Kinesiology, University of British Columbia, Vancouver, BC, Canada, ${ }^{2}$ Behavioural Medicine Laboratory, School of Exercise Science, Physical and Health Education, University of Victoria, Victoria, BC, Canada, ${ }^{3}$ ParticipACTION, Toronto, ON, Canada, ${ }^{4}$ Child Health and Evaluative Sciences, Hospital for Sick Children, Toronto, ON, Canada, ${ }^{5}$ School of Hospitality, Food and Tourism Management, University of Guelph, Guelph, ON, Canada, ${ }^{6}$ College of Kinesiology, University of Saskatchewan, Saskatoon, SK, Canada, ${ }^{7}$ Faculty of Kinesiology, Sport, and Recreation, University of Alberta, Edmonton, $A B$, Canada

COVID-19 was declared a global pandemic by the World Health Organization on March 11, 2020. The COVID-19 pandemic and resulting social disruption has left many to struggle with changes to routines and feelings of uncertainty as the impact of the virus continues to unfold. Evidence suggests an increase in symptoms of anxiety, depression, and stress as a result. Given the well-documented association between physical activity and mental health - be it preventing the onset of depressive or anxious symptoms to minimizing the prognosis of certain conditions - we posit more emphasis be placed in health communications on physical activity as a coping strategy for Canadians. As the ramifications of COVID-19 continue, coupled with the concern of a pending second wave, identifying how Canadians are managing stress and mental health can inform the development of interventions aimed at mitigating the negative impact of COVID-19 on adults' overall wellness. Though social interactions and activities might look different right now, Canadians should be actively looking for safe ways to engage in health promoting and socializing behaviors - physical activity is one such behavior. Herein we explore how a representative sample of Canadian adults are coping with increased stress and mental health issues as a result of COVID-19 and identify a potential disconnect between considering physical activity as a strategy to support social connection and stress management and how Canadians are coping with the pandemic. Given the protective role of physical activity in supporting mental health, our perspective is that health communication efforts should focus on the mental health benefits of physical activity particularly during these uncertain times.

Keywords: COVID, physical activity, mental health, social marketing, health communication 


\section{INTRODUCTION}

COVID-19 was declared a global pandemic by the World Health Organization on March 11, 2020 (World Health Organization, 2020). In response to the fast-spreading virus, states of emergency were declared worldwide resulting in communitywide lockdowns and "stay-at-home" orders (Dawson, 2020). Countries implemented lockdowns and social distancing regulations at differing rates and levels, but by early April 2020, more than $90 \%$ of the world's population were under some level of restriction (Connor, 2020). Initially, these COVID-19related closures and restrictions undoubtedly disrupted daily routines, arrangements, and rhythms of individual and family lives. As a result, the COVID-19 pandemic has negatively impacted mental health globally. Emerging literature among adults indicates a $16-28 \%$ increase in anxiety and depressive symptoms, and a $5 \%$ increase in self-reported stress since the onset of the pandemic (Rajkumar, 2020). Contributing issues include social isolation, travel bans, border closures, concerns about personal or familial health, financial constraints, job loss, and interruptions to education. Importantly, the impact of these issues is extensive and far-reaching, and have left many Canadians, at least anecdotally, feeling stressed, anxious, depressed, scared, and bored during these unprecedented times (Canadian Mental Health Association, 2020; Centre for Addictions Mental Health, 2020).

Various coping strategies have been identified in the literature as being effective in addressing mental health, including pharmaceuticals (Gartlehner et al., 2017), psychotherapy, brightlight therapy (Holvast et al., 2017), and meditation (Jain et al., 2015). Physical activity has also been highlighted in the literature as an important modifiable lifestyle behavior for brain function and development (Sharma et al., 2006; American Psychological Association, 2011; Chekroud et al., 2018). Specific to mental health, there is evidence reporting a positive association between physical activity (particularly at higher intensities) and alleviation of symptoms related to anxiety and depression (Sharma et al., 2006; Chekroud et al., 2018; Schuch et al., 2018; Schuch and Stubbs, 2019). The use of physical activity to treat and/or prevent mental health disorders has met with increasing clinical and scientific interest, due to lower side-effect burden, increased accessibility and health promoting abilities, as well as potential reduction of polypharmacy. The mechanisms behind such phenomena are varied including plausible neurobiological, psychosocial, and behavioral mechanisms with the most popular theories being linked to the propagation of neurotransmitters (i.e., dopamine, serotonin, glutamate, GABA) (Maddock et al., 2016) and neurotrophins (BDNF) (Schuch et al., 2016), as well as an increase in neurogenesis, synaptogenesis, and angiogenesis (Saraulli et al., 2017). There is also a body of work to suggest that physical activity can assist with reducing stress reactivity (Mücke et al., 2018) and enhancing resiliency (Childs and de Wit, 2014).

\section{HOW ARE CANADIANS COPING NOW?}

Though social interactions and activities might look different right now, Canadians should be actively looking for safe ways to engage in health promoting and socializing behaviors-physical activity is one such behavior. In April 2020, ParticipACTION (www.participaction.com), a national non-profit organization tasked with educating, advocating, and providing opportunities for Canadians to be more physically active and less sedentary, launched a large-scale brand survey to explore how Canadian adults are coping with increased stress and mental health issues as a result of the COVID-19 pandemic. A representative sample of 1,500 Canadian adults (18+) were invited to complete a 15-min online survey in either French or English over a 7 days period in April 2020. Participants who completed the survey received small cash incentives valued at $\$ 0.50-\$ 3.00$. The sample was comparable to the Canadian census in terms of age, gender, geography, income, and language spoken. Questions included assessment of self-reported levels of physical activity and attitudes toward coping mechanisms for stress, anxiety, and social isolation. Demographic information (i.e., age, gender, education, marital status, and household income) was also collected. Participants were recruited by Maru/Matchbox, a third-party market research company. Maru/Matchbox panelists consent to participate in survey-based research when they sign-up for the panel and when they agreed to complete this survey.

Approximately half the participants were male (49.0\%), with a mean age of 48 years $(S D=15.4)$, the majority had a postsecondary degree $(85 \%)$, and $60 \%$ reported an annual household income $<\$ 100,000$. Upon examination of responses provided regarding mental health coping behaviors, it was revealed that only $48 \%$ engaged in physical activity, which was lower than screen-use behaviors (54\%), but more than prescribed medication (14\%) or virtual consults with a therapist or physician $(8 \%)$. Only $1 \%$ of the sample reported that nothing helped with their mental health (see Table 1). Cross-tabulations indicated that younger Canadians (56\% among those 18-34 years vs. $43 \%$ among those $55+$ years), as well as more educated (54\% with university degrees vs. $37 \%$ with high school education or less) and higher income earners ( $\$ 100 \mathrm{~K}+$ /year vs. $<\$ 50 \mathrm{~K} /$ year $)$, were significantly more likely to be engaging in physical activity to cope with their mental health $(p<0.05)$. No genderbased differences were ascertained $(p=0.13)$. Table 2 provides a list of various behaviors on which respondents indicated placing more/less emphasis while social distancing. Compared to physical activity (48\%), $\sim 35$ and $36 \%$ reported placing more emphasis on connecting with family and friends as well as relaxing and "de-stressing." Twenty percent of Canadians reported placing less emphasis on physical activity during the pandemic - the greatest "de-emphasis" compared to all other behavior choice options.

\section{PERSPECTIVE}

Considering global declines in physical activity (Fitbit, 2020), we were interested in understanding how Canadians coped throughout COVID-19 and the role of physical activity in supporting mental health during the pandemic. Data collected by ParticipACTION suggests Canadians are less likely to use 
TABLE 1 | Mental health coping behaviors among Canadians during COVID-19 $(n=1,472)$.

Behavior type

Percent of adults

Watch TV/Movies (this could include Netflix, Crave, Amazon Prime, etc.)

Physical activity

Favorite hobbies (gaming, crafts, checking social media etc.)

Spending time with family/friends

Relaxation (bath, hot water bottle, etc.)

Alcohol

Yoga/stretching

Having a cry

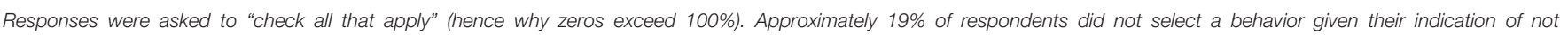
experiencing depression/anxiety.

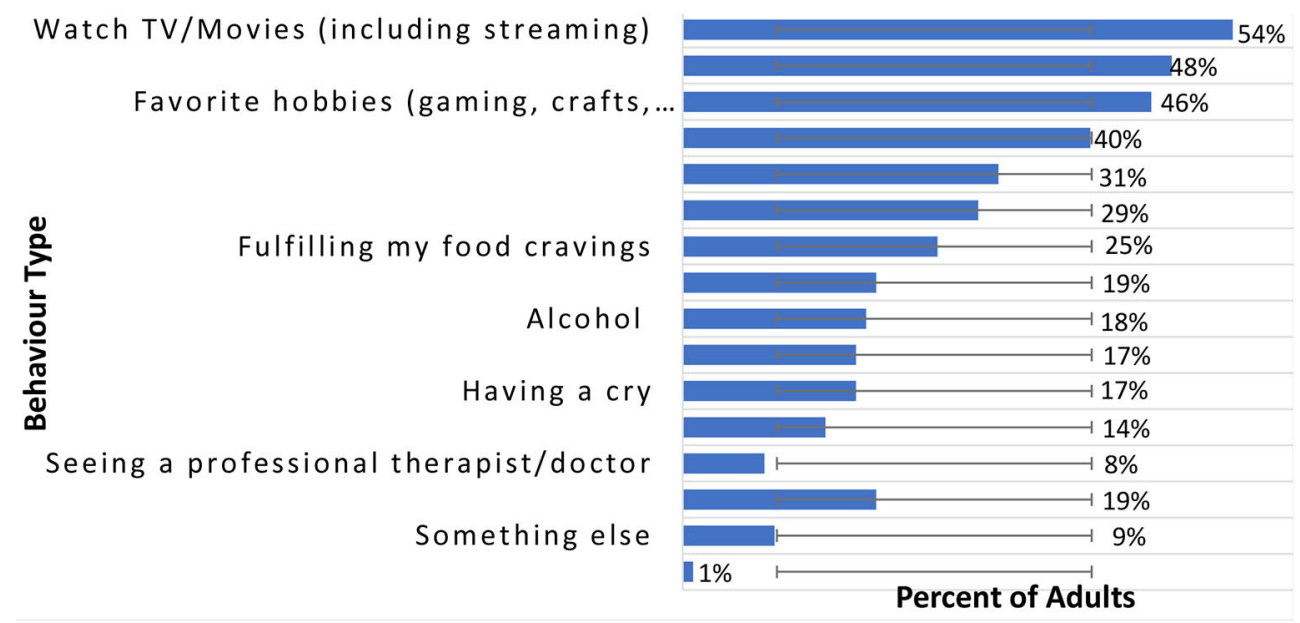

physical activity as compared to more sedentary activities (e.g., watching TV) as a coping mechanism for stress or anxiety reduction. Given broad "stay-at-home" directions, it might be expected that individuals are more likely to resort to passive indoor leisure vs. active leisure to handle stress in generalpandemic or not. More concerning is a potential reluctance to consider physical activity as a primary coping measure. This risks shifting an already inactive population even lower.

Correlates of using physical activity as a coping mechanism are consistent with prior research-younger, more educated, and those of higher socio-economic status (Cairney et al., 2014). These individuals may have better access to physical activity resources (whether online or in their personal homes) and more time (e.g., fewer family responsibilities). Further, during times of social isolation, Canadians are reporting placing less emphasis on engaging in physical activity compared to connecting with family or friends and relaxing/distressing. The current COVID19 crisis has created a "new normal," with less emphasis placed on physical activity (in comparison to screen use, socialization) by a significant minority of Canadians. The fact that $20 \%$ of respondents reported less emphasis on physical activity during the pandemic suggests that the social distancing and isolation regulations may have put new barriers in place for some to engage in physical activity compared to pre-pandemic times.

The findings suggest a potential disconnect between participating in physical activity as a strategy to support social connection and stress management and how Canadians are coping with stress in particular. While it is possible that people are choosing less effective strategies to deal with the consequences of COVID-19 (possibly due to personal interest or boredom), it is important that individuals remain informed of the compounding effects behaviors like excess screen-viewing can have on the mind and body, potentially exacerbating symptoms of poor mental health (Madhav et al., 2017). Consequently, we believe a potential role exists for social marketing initiatives to address this disconnect and communicate more explicitly the mental health 
TABLE 2 | Level of emphasis placed by Canadian adults while social distancing during COVID-19 ( $n=1,472)$.

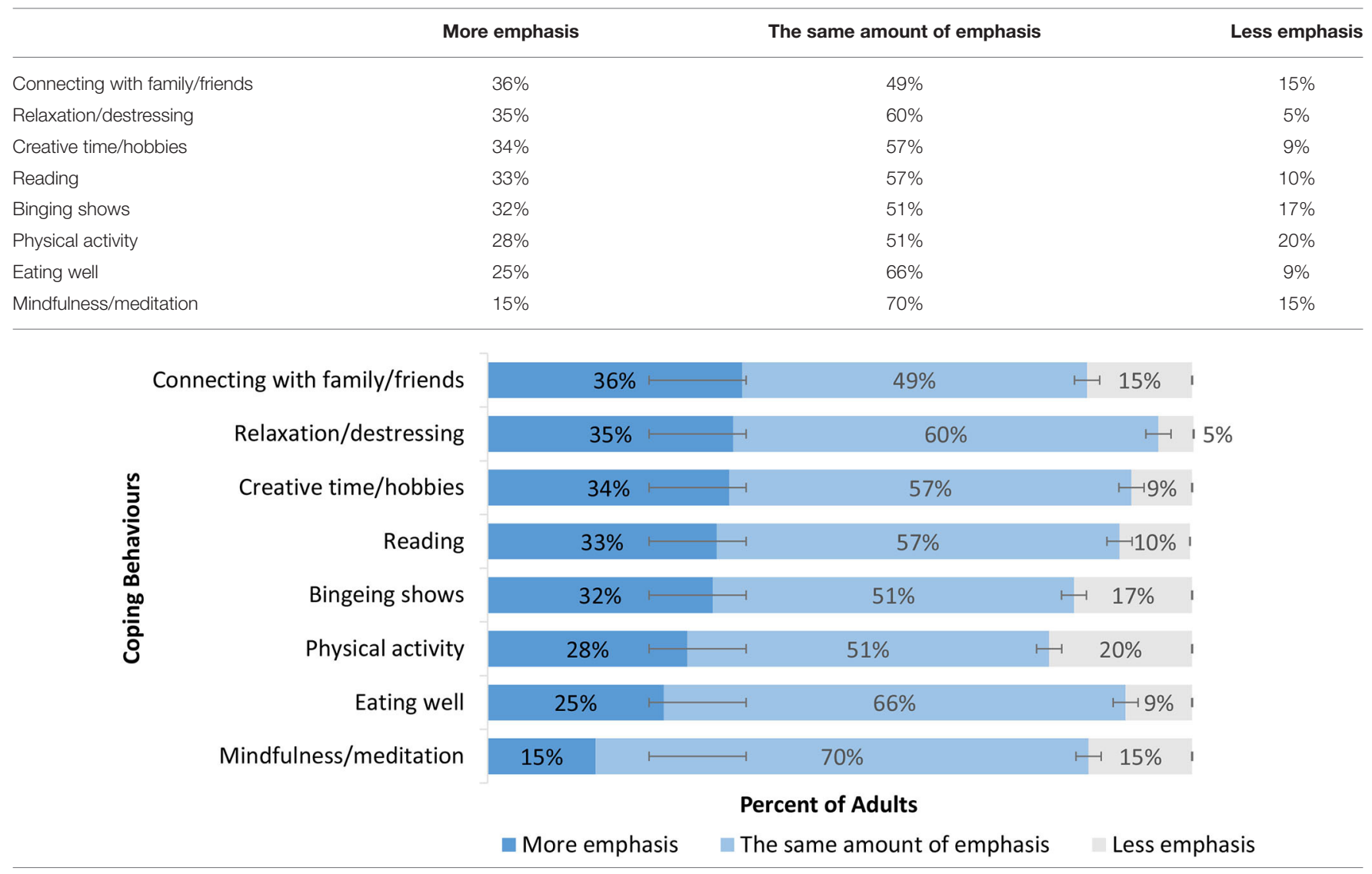

benefits of physical activity. In the context of physical distancing measures, this may take the form of focusing on family or household co-participation in physical activity. Sharing evidence about the stress management benefits of physical activity may also be beneficial. Such messaging also needs to convey the "how to" and develop individual's self-efficacy to engage in physical activity for mental health benefits. Consequently, health communications are likely to be less than optimally beneficial if they are not supported by parallel initiatives that support access to opportunities for physical activity while allowing for physical distancing (Institute of Medicine, 2015). For example, these initiatives could include temporary reallocation of roadway space and keeping expansive green spaces open. In dense urban environments, initiatives could include reallocating traffic lanes to allow for cycling and walking with social distancing possible.

In response to the COVID-19 pandemic, coupled with the growing incidence of stress, anxiety, and depression among Canadians (Rajkumar, 2020), many other health-based organizations have begun to tailor their communication and marketing efforts to better reflect the current social and environmental climate. For example, based on the presented results ParticipACTION's social marketing and communications platforms will now focus on educating Canadians on how engaging in physical activity can support social connectivity and mental health. By strategically re-working the organization's content plans (including key messages, web and e-communications messaging, app content, and marketing materials), ParticipACTION aims to facilitate physical activity participation as a coping mechanism during stressful life situations. ParticipACTION plans to develop content, resources and opportunities for Canadians to be more active where they live, work and play. Through ongoing evaluations, ParticipACTION will assess whether the organization's new focus on social wellness and mental health reduces the disconnect between coping with stress and using physical activity as a tool to manage stress.

We provide data suggesting evidence of immediate collateral consequences of the COVID-19 outbreak with regards to how Canadian adults are coping with stress and mental health as well as the reduced role of physical activity in addressing or supporting these issues. For the immediate future, organizations like ParticipACTION are uniquely positioned during this pandemic to re-tool its messaging and engagement initiatives to help encourage Canadians to participate in more physical activity. As the ramifications of COVID-19 continue, coupled with the concern of a pending second wave, supporting Canadians to better manage stress and wellness by encouraging physical activity participation may help to mitigate the negative impact of the virus and related social distancing on adults' mental health. We suggest that physical activity is an important 
health behavior worth highlighting when developing health communications and social marketing campaigns specific to supporting social connectedness and mental health during the current pandemic.

\section{DATA AVAILABILITY STATEMENT}

The raw data supporting the conclusions of this article will be made available by the authors, without undue reservation.

\section{ETHICS STATEMENT}

Ethical review and approval was not required for the study on human participants in accordance with the local legislation and institutional requirements. The patients/participants provided their written informed consent to participate in this study.

\section{REFERENCES}

American Psychological Association. (2011). The exercise effect. Am. Psychol, Assoc. 42:48.

Cairney, J., Kwan, M. Y., Veldhuizen, S., and Faulkner, G. E. (2014). Who uses exercise as a coping strategy for stress? results from a national survey of Canadians. J. Phys. Act Heal. 11, 908-916. doi: 10.1123/jpah.2012-0107

Canadian Mental Health Association. (2020). Canadians Are Anxious and Crave Real Connection, but Say They're Doing "Fine". Available online at: https://cmha.ca/news/canadians-are-anxious-and-crave-real-connectionbut-say-theyre-doing-fine (accessed June 12, 2020)

Centre for Addictions and Mental Health. (2020). COVID-19 National Survey Dashboard. Available online at: http://www.camh.ca/en/health-info/mentalhealth-and-covid-19/covid-19-national-survey (accessed June 12, 2020)

Chekroud, S. R., Gueorguieva, R., Zheutlin, A. B., Paulus, M., Krumholz, H. M., Krystal, J. H., et al. (2018). Association between physical exercise and mental health in 1.2 million individuals in the USA between 2011 and 2015: a cross-sectional study. Lancet Psychiatry 5, 739-746. doi: 10.1016/S2215-0366(18)30227-X

Childs, E., and de Wit, H. (2014). Regular exercise is associated with emotional resilience to acute stress in healthy adults. Front. Physiol. 5:161. doi: 10.3389/fphys.2014.00161

Connor, P. (2020). More Than Nine-In-Ten People Worldwide Live in Countries With Travel Restrictions Amid COVID-19. Pew Research Center. Available online at: https://www.pewresearch.org/fact-tank/2020/04/01/more-thannine-in-ten-people-worldwide-live-in-countries-with-travel-restrictionsamid-covid-19/ (accessed Jun 8, 2020).

Dawson, T. (2020). As the COVID-19 Pandemic Hit, Provinces Declared States of Emergency. Now Many Are Up for Renewal. Available online at: https:// nationalpost.com/news/provincial-states- of-emergencies-were-issued-amonth-ago-most-are-coming-up-for-renewal (accessed June 12, 2020)

Fitbit. (2020). Fitbit News. Available online at: https://blog.fitbit.com/covid-19global-activity/ (accessed June 12,2020 ).

Gartlehner, G., Wagner, G., Matyas, N., Titscher, V., Greimel, J., Lux, L., et al. (2017). Pharmacological and non-pharmacological treatments for major depressive disorder: review of systematic reviews. BMJ Open. 7:e014912. doi: 10.1136/bmjopen-2016-014912

Holvast, F., Massoudi, B., Oude Voshaar, R. C., and Verhaak, P. F. M. (2017). Non-pharmacological treatment for depressed older patients in primary care: a systematic review and meta-analysis. PLoS ONE. 12:e0184666. doi: 10.1371/journal.pone.0184666

Institute of Medicine. (2015). Communicating to Advance the Public's Health. Effective Messaging Strategies: A Review of the Evidence. Washington, DC: Institute of Medicine.

Jain, F. A., Walsh, R. N., Eisendrath, S. J., Christensen, S., and Rael Cahn, B. (2015). Critical analysis of the efficacy of meditation therapies for acute and subacute phase treatment of depressive disorders: a systematic review. Psychosomatics 56, 140-152. doi: 10.1016/j.psym.2014.10.007

\section{AUTHOR CONTRIBUTIONS}

GF, TC-B, and LV conceptualized the manuscript, analyzed, interpreted the available data, and drafted the initial draft of the manuscript. All authors read, reviewed, and approved the final version of the paper.

\section{FUNDING}

LV holds a Canadian Institutes of Health Research (CIHR) Fellowship Award. GF holds a CIHR-Public Health Agency of Canada Chair in Applied Public Health.

\section{ACKNOWLEDGMENTS}

We would like to thank the survey participants for their support of the project.

Maddock, R. J., Casazza, G. A., Fernandez, D. H., and Maddock, M. I. (2016). Acute modulation of cortical glutamate and GABA content by physical activity. J. Neurosci. 36, 2449-2457. doi: 10.1523/JNEUROSCI.3455-1 5.2016

Madhav, K. C., Sherchand, S. P., and Sherchan, S. (2017). Association between screen time and depression among US adults. Prev. Med. Rep. 8, 67-71. doi: 10.1016/j.pmedr.2017.08.005

Mücke, M., Ludyga, S., Colledge, F., and Gerber, M. (2018). Influence of regular physical activity and fitness on stress reactivity as measured with the trier social stress test protocol: a systematic review. Sport Med. 48, 2607-2622. doi: 10.1007/s40279-018-0979-0

Rajkumar, R. P. (2020). COVID-19 and mental health: a review of the existing literature. Asian J. Psychiatr. 52:102066. doi: 10.1016/j.ajp.2020.102066

Saraulli, D., Costanzi, M., Mastrorilli, V., and Farioli-Vecchioli, S. (2017). The long run: neuroprotective effects of physical exercise on adult neurogenesis from youth to old age. Curr. Neuropharmacol. 15, 519-533. doi: 10.2174/1570159X14666160412150223

Schuch, F. B., Dunn, A. L., Kanitz, A. C., Delevatti, R. S., and Fleck, M. P. (2016). Moderators of response in exercise treatment for depression: a systematic review. J. Affect Disord. 195, 40-49. doi: 10.1016/j.jad.2016.01.014

Schuch, F. B., and Stubbs, B. (2019). The role of exercise in preventing and treating depression. Curr. Sports Med. Rep. 18, 299-304. doi: 10.1249/JSR.0000000000000620

Schuch, F. B., Vancampfort, D., Firth, J., Rosenbaum, S., Ward, P. B., Silva, E. S., et al. (2018). Physical activity and incident depression: a metaanalysis of prospective cohort studies. Am. J. Psychiatry. 175, 631-648. doi: 10.1176/appi.ajp.2018.17111194

Sharma, A., Madaan, V., and Petty, F. D. (2006). Exercise for mental health. Prim. Care Companion J. Clin. Psychiatry 8:106. doi: 10.4088/PCC.v08n 0208a

World Health Organization (2020). Coronavirus Disease (COVID-19) Pandemic: WHO Characterizes COVID-19 as a Pandemic. Geneva.

Conflict of Interest: GF, JS, NO'R, RR, and LF are members of the ParticipACTION Research Advisory Group (RAG); a panel of experts that provides strategic direction regarding the organization's research, evaluation, and knowledge translation activities. ParticipACTION provides meeting expenses for the RAG to meet but does not provide any additional compensation. TC-B and LV are employed by ParticipACTION in the roles of Behavioral Insights Manager and Knowledge Translation Manager, respectively.

Copyright (c) 2020 Faulkner, Rhodes, Vanderloo, Chulak-Bozer, O’Reilly, Ferguson and Spence. This is an open-access article distributed under the terms of the Creative Commons Attribution License (CC BY). The use, distribution or reproduction in other forums is permitted, provided the original author(s) and the copyright owner(s) are credited and that the original publication in this journal is cited, in accordance with accepted academic practice. No use, distribution or reproduction is permitted which does not comply with these terms. 